\title{
The Inflammatory Response Induced by RELM $\beta$ Upregulates IL-8 and IL-I $\beta$ Expression in Bronchial Epithelial Cells in COPD
}

\author{
Li Che $\mathbb{D}^{\prime}$ \\ Chao $\mathrm{Yu}^{2}$ \\ Guangshu Chen ${ }^{3}$ \\ Jiaxin Lin' \\ Zhefan Xie' \\ Tingting $\mathrm{Xia}^{\prime}$ \\ Wenzhi Luo' \\ Xingdong Cai $\mathbb{D}^{\prime}$ \\ Shengming Liu' \\ 'Department of Pulmonary and Critical \\ Care Medicine, The First Affiliated \\ Hospital of Jinan University, Guangzhou, \\ 510630, People's Republic of China; \\ ${ }^{2}$ Department of Pulmonary and Critical \\ Care Medicine, Lu'an People's Hospital of \\ Anhui Province, Lu'an, 237016, People's \\ Republic of China; ${ }^{3}$ Department of \\ Endocrinology, Guangzhou Red Cross \\ Hospital, The Affiliated Hospital of Jinan \\ University, Guangzhou, 510220, People's \\ Republic of China
}

Purpose: Chronic obstructive pulmonary disease (COPD) is associated with a complex inflammatory regulatory network. Resistin-like molecule $\beta$ (RELM $\beta)$ is highly expressed in the lungs of COPD patients. We aimed to investigate the proinflammatory effect of RELM $\beta$ on airway epithelial cells in COPD.

Methods: First, a GEO dataset was used to analyze the expression of the RELM $\beta$ gene in the COPD and control groups as well as the protein levels of RELM $\beta$ in the sera of outpatients with COPD and normal control subjects in our hospital. We also stimulated $16 \mathrm{HBE}$ bronchial epithelial cells with recombinant RELM $\beta$ protein and analyzed the expression of IL- 8 and IL-1 $\beta$. We upregulated and downregulated the gene expression of RELM $\beta$ in $16 \mathrm{HBE}$ cells and analyzed the expression of the inflammatory cytokines IL- 8 and IL-1 $\beta$. In addition, we also examined the mechanism by which the p38 MAPK signaling pathway contributed to the regulation of IL- 8 and IL- $1 \beta$ expression by RELM $\beta$.

Results: RELM $\beta$ expression was increased in COPD tissues in different data sets and in the serum of COPD patients in our hospital. IL- 8 and IL-1 $\beta$ expression was also increased in COPD tissues with high RELM $\beta$ gene expression in different data sets. The RELM $\beta$ gene was mainly related to inflammatory factors and inflammatory signaling pathways in the PPI regulatory network. Experiments at the cellular level showed that RELM $\beta$ promoted the expression of the inflammatory cytokines IL-8 and IL-1 $\beta$, and this regulation was mediated by the p38 MAPK signaling pathway.

Conclusion: RELM $\beta$ can promote the expression of the inflammatory cytokines IL- 8 and IL-1 $\beta$ in bronchial epithelial cells of patients with COPD and exert inflammatory effects. RELM $\beta$ may be a potential target for the treatment of COPD.

Keywords: resistin-like molecule beta/RELM $\beta$, chronic obstructive pulmonary disease, p38 MAPK, IL-8, IL-1 $\beta$

\section{Introduction}

The prevalence of chronic obstructive pulmonary disease (COPD) in China is $8.6 \%$ in people over 20 years of age and $13.7 \%$ in people over 40 years of age, and there

Correspondence: Shengming Liu; Xingdong Cai

Department of Pulmonary and Critical Care Medicine, The First Affiliated

Hospital of Jinan University, The West of Huangpu Road 613, Tianhe District,

Guangzhou, Guangdong, People's

Republic of China

Tel +862038688628

Email tlsm@jnu.edu.cn;

cxd19790920@sina.com are approximately 100 million COPD patients in China; ${ }^{1}$ in addition, the prevalence of COPD is increasing. ${ }^{2}$ Although new drugs are constantly entering the clinic to benefit more patients, the disease burden of patients with COPD is still increasing. ${ }^{3}$ Approaches for inhibiting the progression of COPD are unclear. Airway inflammation likely dominates the pathogenesis and progression of COPD. ${ }^{4}$ The pathological changes associated with COPD include repeated airway inflammatory infiltration, 
inflammatory cell accumulation in different parts of the lung, and oxidative stress resulting from slight airway injury and lung parenchyma destruction. Subsequently, pathological changes in COPD include airflow limitation, abnormal gas exchange, and decreased lung function. ${ }^{5-8}$ Therefore, an in-depth study of the specific mechanisms underlying airway inflammation in COPD is of great significance for the diagnosis and treatment of COPD.

RELM $\beta$ also known as found in the inflammatory zone family (FIZZ2), is specifically expressed in human lung tissue, promotes lung cell mitosis and proliferation, and participates in inflammatory and immune responses. ${ }^{7}$ RELM $\beta$ plays an essential role in pulmonary diseases, such as pulmonary hypertension, asthma, and pulmonary fibrosis. $^{9-11}$ Hypoxia and inflammation are among the main causes of the high expression of RELM $\beta$ in airways. $^{12}$ It has been shown that RELM $\beta$ is highly expressed in lung tissues of patients with hypoxiamediated pulmonary hypertension and is observed in remodeled vascular endothelial cells, vascular smooth muscle cells, macrophages and fibroblasts. ${ }^{13}$ In addition, RELM $\beta$ overexpression in mice causes hemodynamic changes, including increased mean proper ventricular systolic pressure, right heart hypertrophy, and small pulmonary artery constriction. ${ }^{14}$ The current understanding of the mechanism by which RELM $\beta$ contributes to pulmonary inflammation is mainly derived from studies of RELM $\alpha$, which is highly homologous to RELM $\beta .{ }^{15}$ In a study of rat alveolar epithelial cell inflammation in response to tobacco smoke extract, IL-8 secretion into the supernatant was correlated with RELM $\alpha$ expression in a time-dependent manner. ${ }^{15}$ Studies by Yamaji-Kegan et al showed that IL-4 expression was significantly elevated in the lungs of wild mice treated with RELM $\alpha$, while RELM $\alpha$ treatment of pulmonary microvascular endothelial cells stimulated cell proliferation, and that this process was dependent on the IL4/IL-4R $\alpha$ system. ${ }^{12}$ In addition, some investigators observed that both RELM $\alpha$ and RELM $\beta$ increased IL-6 expression in lung epithelial cells and increased the perivascular aggregation of IL-6-expressing macrophages in mouse lungs. ${ }^{16}$ These findings suggest that RELM $\alpha$ plays an essential role in mediating chronic pulmonary inflammation. However, the role of RELM $\beta$ in chronic airway inflammation has been poorly studied.

In our study, we observed that RELM $\beta$ mRNA expression was increased in patients with COPD compared with normal individuals. Therefore, we hypothesized that the RELM $\beta$ gene might be an essential regulatory factor in the chronic inflammatory network of COPD patients. We observed the expression of inflammatory cytokines after up- or downregulating RELM $\beta$ gene expression in bronchial epithelial cells to test our hypothesis. The experimental findings suggest that RELM $\beta$ can promote the expression of inflammatory cytokines.

\section{Materials and Methods}

\section{Transcriptional Expression Profile and Patients from the GEO Database}

We obtained RELM $\beta$ mRNA expression data from the Gene Expression Omnibus (GEO) database (http://www.ncbi.nlm. nih.gov/geo). We screened two datasets, GSE106986 and GSE56766, by searching for differential RELM $\beta$ mRNA expression in patients with COPD from the GPL570 microarray platform. We further analyzed the differential RELM $\beta$ mRNA expression in control and COPD tissues in these two different datasets by probe ID conversion. In addition, we analyzed the differences in the high and low mRNA expression of RELM $\beta$ and the expression of the inflammatory factors IL-8 and IL-1 $\beta$ in different microarray platforms (platform number GPL13243, GSE37147; platform number GPL570, GSE 56766). Serum was obtained from 20 control subjects and 27 patients with COPD, according to the Global Initiative for Chronic Obstructive Lung Disease criteria. ${ }^{1}$

\section{Protein-Protein Interactions (PPIs) Network Construction and Enrichment Analysis}

The PPIs network analysis is a prediction of the genes associated with the molecule for the purpose of analysis of previous studies and on the basis of previous studies. The PPIs were obtained using the Search Tool for the Retrieval of Interacting Genes (STRING; http://string-db.org) online database. Gene Ontology (GO) and Kyoto Encyclopedia of Genes and Genomes (KEGG) signal pathway enrichment analyses of RELM $\beta$ and inflammatory factors were conducted with Cytoscape (V.3.6.0) software. We acquired the enrichment analysis maps from the online data analysis website (http://www.bioinformatics.com.cn/).

\section{Cell Lines and Transfection of Small Interfering RNA}

Human 16HBE bronchial epithelial cells were obtained from Sun Yat-sen University. The cell line was cultured using highglucose DMEM containing $10 \% \mathrm{FBS}$ at $37^{\circ} \mathrm{C}$ in a $5 \% \mathrm{CO} 2$ 
thermostatic cell culture incubator. The cells were cultured using antibiotic-free medium and plated in 6-well plates to ensure that the cell confluence for transfection was approximately $50-70 \%$ after $24 \mathrm{~h}$. Transfection reagent LipoFectMax ${ }^{\mathrm{TM}}$ (ABP Bioscience, Wuhan, China) and diluted RELM $\beta$ small interfering RNA (siRNA) (GenePharma, Shanghai, China) were added to the Opti-MEM transfection medium at a specific ratio. The transfection mixture was discarded after six hours and replaced with completely fresh medium, and the cells were cultured and tested after $24-48 \mathrm{~h}$.

\section{Reverse Transcription-PCR (RT-PCR) and Real-Time Quantitative PCR (Q-PCR)}

Total RNA was extracted from bronchial epithelial cells using TRIzol reagent (Invitrogen, San Diego, CA) and reverse transcribed into cDNA using a reverse transcription kit (TsingKe, China). The PCR amplification reaction was then performed. The amplification conditions were $98^{\circ} \mathrm{C}$ for $3 \mathrm{~min}$ and annealing at $60^{\circ} \mathrm{C}$ for $10-15 \mathrm{~s}$, and the products were collected after $30-35$ cycles. Then, the products were separate by agarose gel horizontal electrophoresis. GAPDH was used as the loading control. cDNAs were amplified by quantitative PCR using a CFX instrument (BioRad, Hercules, CA).

\section{ELISA and Western Blotting Analysis}

Cell supernatants were used for ELISA. The supernatants were harvested, and $100 \mu \mathrm{L}$ of antibody dilutions (RELM $\beta$, Abcam, Cambridge, UK; IL-1 $\beta$, Abcam, Cambridge, UK; IL-8, Abcam, Cambridge, UK) were added to each well and incubated for $1 \mathrm{~h}$. The secondary antibodies were added, and the reaction was terminated with the termination solution. A wavelength of $450 \mathrm{~nm}$ was used to measure the absorbance.
For the Western blotting assay, after the total protein concentration was determined, we separated equal amounts of proteins from each well by vertical electrophoresis using SDS-PAGE. We transferred the proteins to polyvinylidene fluoride membranes, followed by immunoblotting. IL-8 and IL-1 $\beta$ primary antibodies were purchased from Abcam (Cambridge, UK).

\section{Statistical Analysis}

Data are expressed as the mean \pm s.d. The rest of the data were analyzed using the Kruskal-Wallis test or one-way ANOVA. $\mathrm{P}<0.05$ was considered statistically significant.

\section{Results \\ RELM $\beta$ Expression Was Increased in COPD}

RELM $\beta$, an analog of RELM $\alpha$ in humans, has been primarily studied as an inflammatory factor in gastrointestinal diseases ${ }^{17-19}$ and has been less frequently studied in airway diseases. In the current study, we analyzed RELM $\beta$ expression in the GEO database using bioinformatics methods, and the results are shown in Figure 1. The GEO database is a public platform database. RELM $\beta$ mRNA levels were significantly higher in COPD samples in the microarray datasets GSE106986 and GSE56766. RELM $\beta$, a secretory protein, can be secreted into the blood. We measured the RELM $\beta$ level in the sera of patients with COPD from our outpatient clinic using the ELSA method. The results suggested that serum RELM $\beta$ levels were also higher in 27 patients with COPD than in control subjects (details of which are listed in Table 1).
A

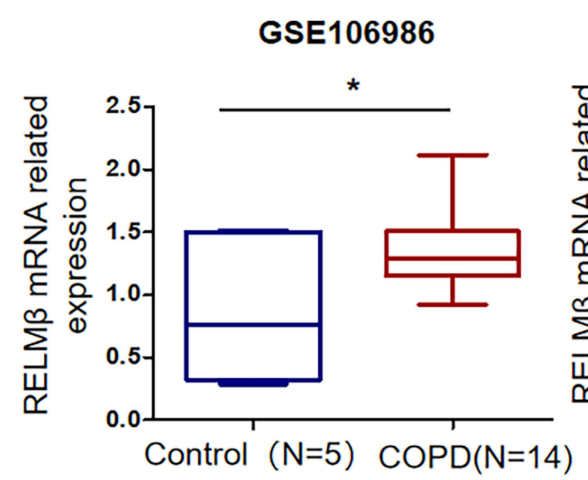

GSE56766

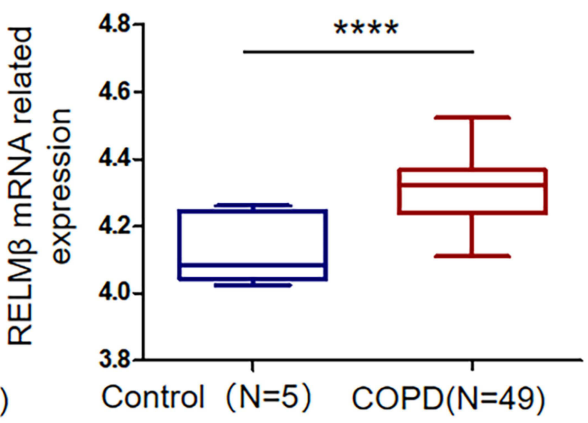

B

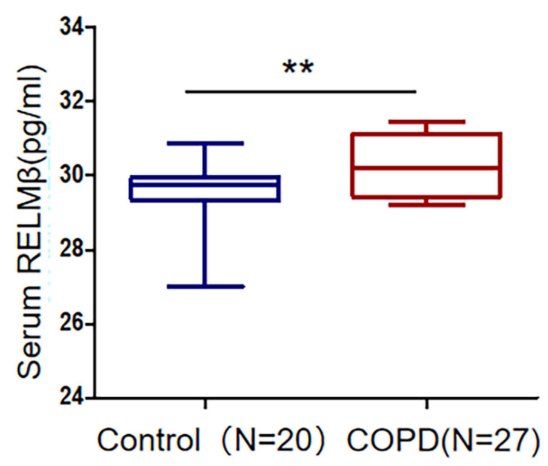

Figure I RELM $\beta$ expression was significantly increased in patients with COPD. (A) RELM $\beta$ mRNA expression in patients with COPD and normal controls in the GEO database. (B) RELM $\beta$ protein expression in the sera of patients with COPD and control individuals. ${ }^{*} \leq 0.05, * * p \leq 0.01, * * * * p \leq 0.0001$. 
Table I Demographics on the Volunteers

\begin{tabular}{|l|c|c|c|}
\hline Disease Group & Age (Y) & Sex (M/F) & Smoking (No/Yes) \\
\hline Control Group & $47-76$ & $17 / 3$ & $15 / 5$ \\
COPD Group & $55-81$ & $21 / 6$ & $14 / 13$ \\
\hline
\end{tabular}

Abbreviation: COPD, chronic obstructive pulmonary disease.

\section{RELM $\beta$ Regulates Inflammatory Networks}

COPD is associated with a complex inflammatory regulation network. ${ }^{20}$ Inflammatory factors exert inflammatory effects and inflammatory amplification effects by recruiting and activating inflammatory cells. We analyzed the inflammatory factors associated with RELM $\beta$ through the STRING online database, and this analysis revealed 16 genes involved in RELM $\beta$ regulation (Figure 2A). The genes associated with RELM $\beta$ comprise the PPIs network indicating that RELM $\beta$ is mainly associated with the regulation of inflammatory factors in disease-related regulation, suggesting that RELM $\beta$ may be mainly involved in inflammatory diseases. We analyzed the possible enriched signaling pathways and gene ontology through an online enrichment analysis tool (http://www.bioinformatics.com. $\underline{\mathrm{cn}}$ ), and the results showed that RELM $\beta$ was related. We show the signal pathway of enrichment analysis top 10 . The results showed that the inflammatory factors associated with RELM $\beta$ regulation were mainly enriched in signaling pathways such as cytokine interaction and JakSTAT. In contrast, the molecular functional enrichment analysis in gene ontology showed that the genes were functionally concentrated in protein linkage and cytokine receptor activation (Figure 2C). Cellular components mainly focused on extracellular regions and extracellular space. Biological processes were mainly involved in regulating immune protein production and the type 2 immune response. The analysis results described above indicated that the inflammatory factors associated with RELM $\beta$ are mainly related to the inflammatory response pathway.

To further analyze the correlation between RELM $\beta$ expression and gene expression of the inflammatory factors mentioned above in COPD, we retrieved the expression data of inflammatory factors in patients in the GSE5676 and GSE37147 datasets of the GEO database. The cytokine IL-8 is an essential neutrophil-associated inflammatory factor in late inflammation. The IL- $1 \beta$ is a more important proinflammatory factor. ${ }^{20}$ We chose IL8 and IL- $1 \beta$ as the subjects of our study. The results showed that in the two different datasets, the expression of the inflammatory factor IL-8 was higher in the high RELM $\beta$ mRNA expression group than in the low RELM $\beta$ mRNA expression group. Similarly, we investigated the mRNA expression of IL- $1 \beta$ in patients with chronic obstructive pulmonary disease, and our results showed higher IL-1 $\beta$ mRNA expression in the high RELM $\beta$ mRNA expression group than in the low RELM $\beta$ mRNA expression group. Our data analysis suggests that RELM $\beta$ may be further involved in regulating the inflammatory factors IL- 8 and IL-1 $\beta$ and regulating airway inflammation in COPD.

\section{RELM $\beta$ Promotes the Expression of the Inflammatory Factors IL-8 and IL- I $\beta$}

The results described above indicate that RELM $\beta$ expression is regulated by a complex network in the context of inflammatory regulatory networks. To further analyze the RELM $\beta$-mediated inflammatory effects, we stimulated in vitro cultured 16HBE bronchial epithelial cells with commercial recombinant human RELM $\beta$ protein (rhRELM $\beta$ ). After stimulation with increasing concentrations of rhRELM $\beta$, the expression levels of the inflammatory factors IL-8 and IL-1 $\beta$ gradually increased (Figure 3A1 and A2). Moreover, the ELLSA results showed that the IL- 8 and IL-1 $\beta$ protein levels in the cell supernatants increased with the concentration of rhRELM $\beta$ (Figure 3B1 and B2). What's more, RELM $\beta$ and CSE have additive effects on the expression of IL- 8 and IL- $1 \beta$ protein. In addition, we generated a stable strain of $16 \mathrm{HBE}$ cells overexpressing RELM $\beta$. We infected 16HBE cells with a lentiviral plasmid containing pLV.O-RELM $\beta$ and the control plasmid pLV.O. Our results showed that the mRNA and protein levels of RELM $\beta$ were significantly higher in the $16 \mathrm{HBE}$ cell line overexpressing the RELM $\beta$ gene than in the null and parental control cell lines (Figure 3C1-C3). These results also indicated that RELM $\beta$ was stably expressed in 16 HBE cells. Then, we further analyzed, in the case of RELM $\beta$ gene overexpression, the regulation of the inflammatory factors IL- 8 and IL-1 $\beta$. At both the mRNA and protein levels, the expression of the inflammatory factors IL- 8 and IL- $1 \beta$ was significantly elevated in the group overexpressing the RELM $\beta$ gene compared to the parental and null control groups (Figure 3D1-D3). Our results show that RELM $\beta$ promotes the expression of the inflammatory factors IL-8 and IL-1 $\beta$ at the mRNA and protein levels. 


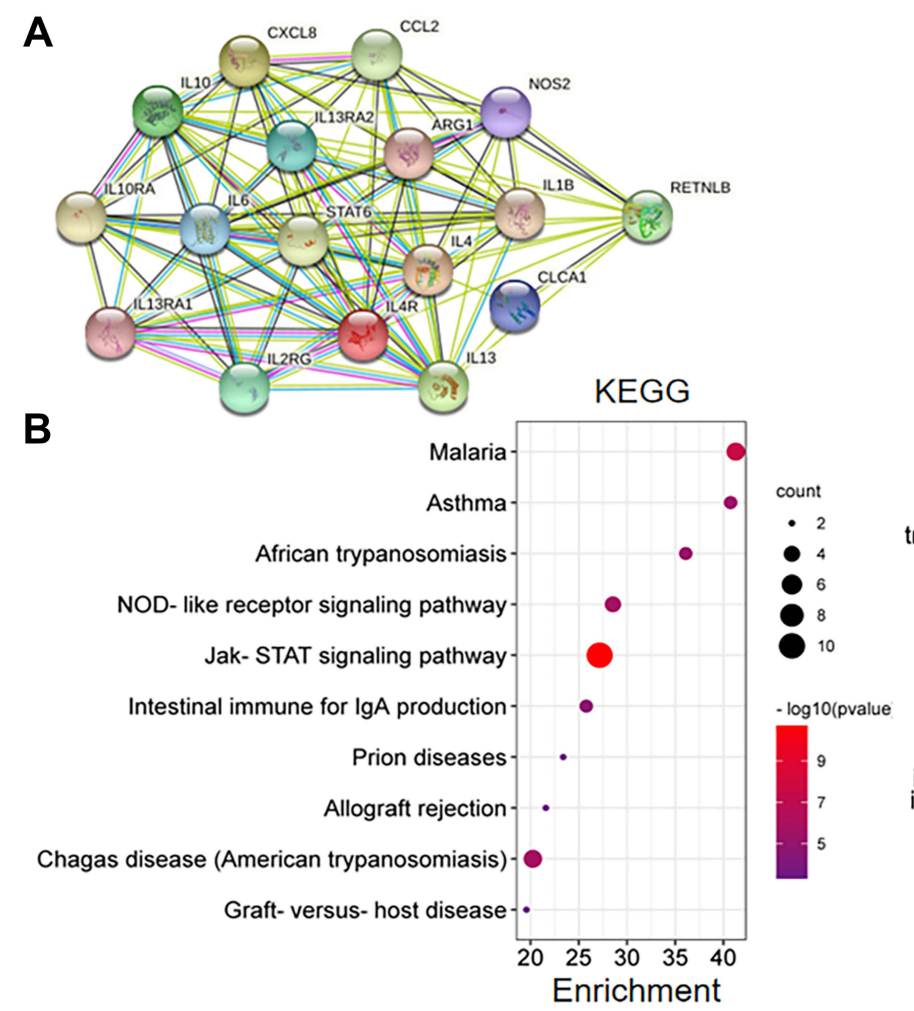

C

GO
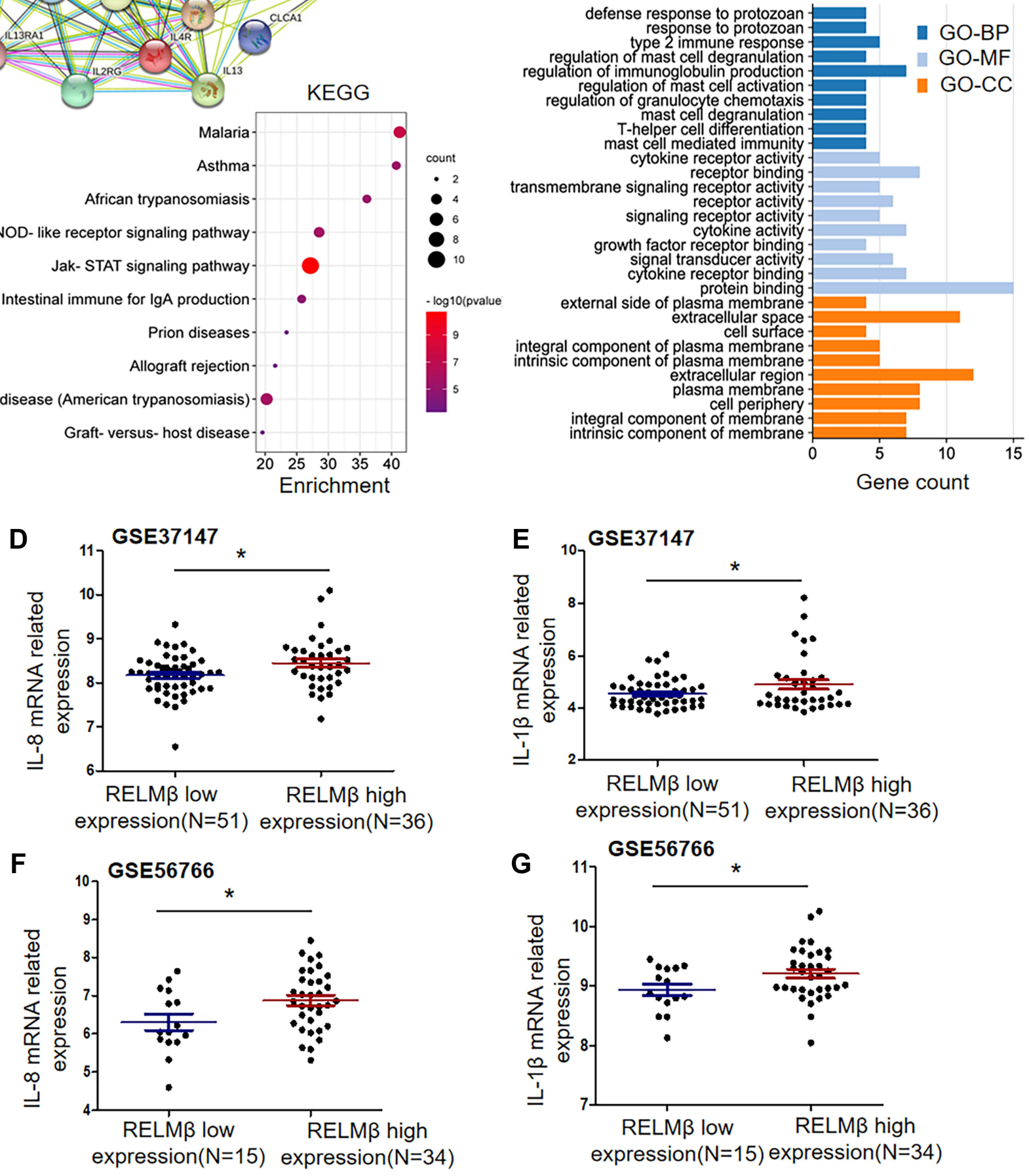

Figure 2 RELM $\beta$ regulates inflammatory networks. (A) The online data analysis site STRING was used to analyze the inflammatory networks involved in the regulation of RELM $\beta$ expression. (B-C) Results of RELM $\beta$ and provocative factor enrichment analysis. (D-G) Correlation of high and low expression of RELM $\beta$ mRNA with the expression of the inflammatory factors IL-8 and IL-I $\beta$. *p $\leq 0.05$.

Abbreviations: $\mathrm{BP}$, biological process; MF, molecular function; $\mathrm{CC}$, cellular component. 

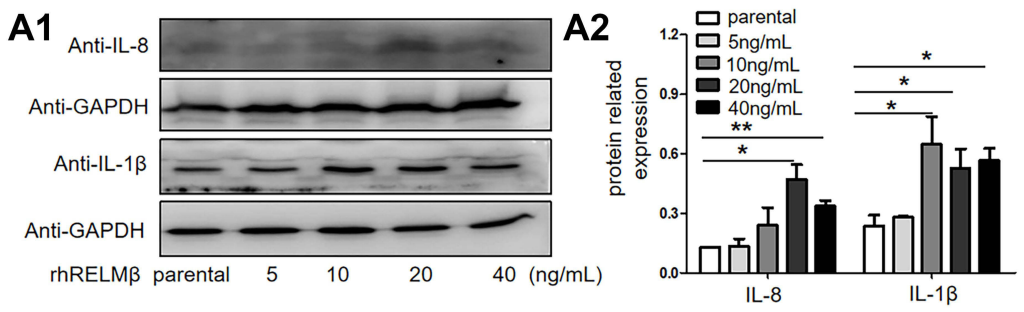

B1

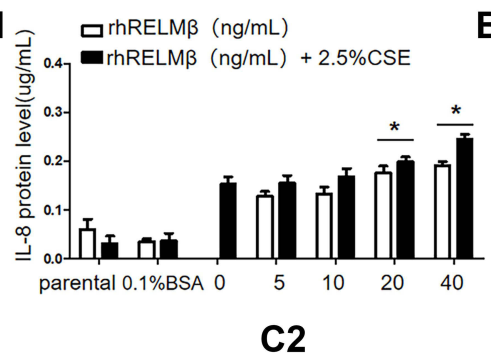

C1

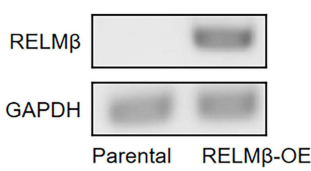

D1
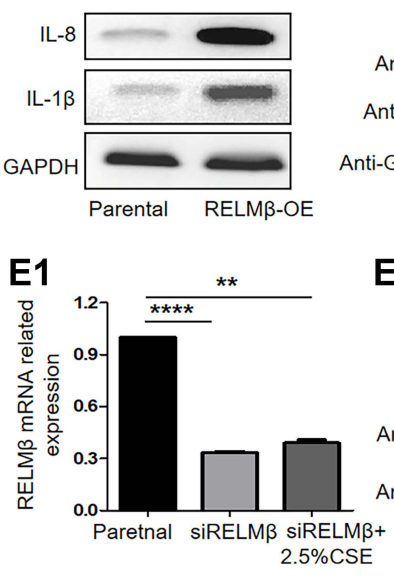

F1

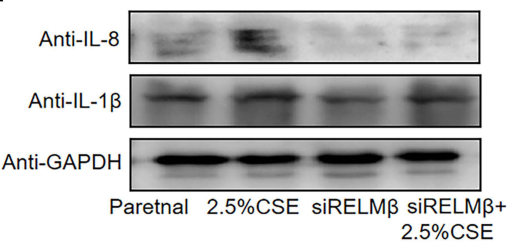

E2
B2

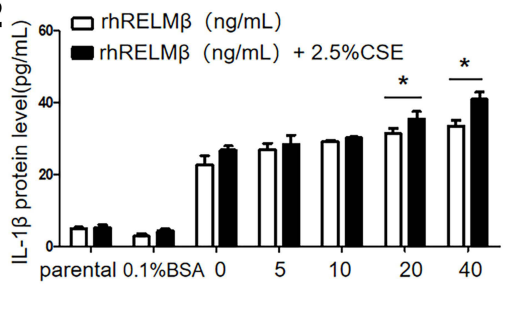

C3

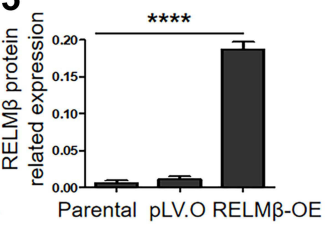

D3

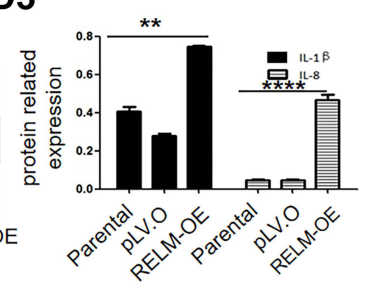

E3

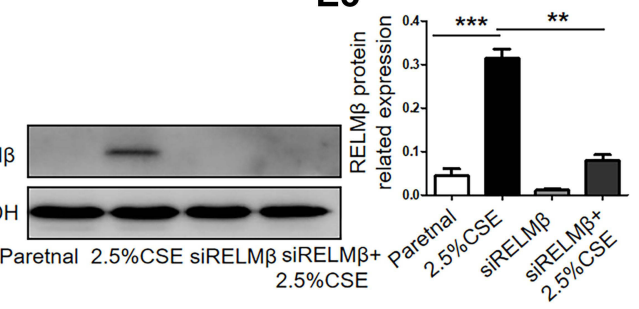

F2

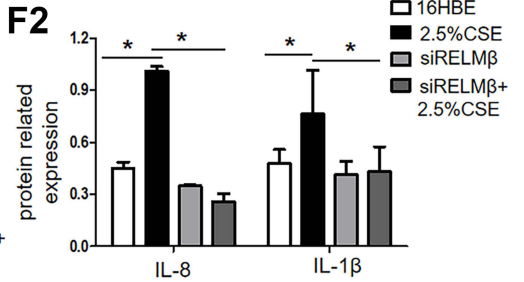

Figure 3 RELM $\beta$ promotes the expression of the inflammatory factors IL-8 and IL-I $\beta$. (AI, A2) I6HBE cells were stimulated with different concentrations of recombinant RELM $\beta$ protein; Western blotting results showed the expression of the inflammatory factors IL-8 and IL-I $\beta$. (BI, B2) ELISA results showed the secretion of the inflammatory factors IL-8 and IL-I $\beta$ into cell supernatants. (CI-C3) Using RT-PCR and Western blotting, we found that RELM $\beta$ expression was increased in I6HBE cells infected with lentiviral particles compared with parental cells and cells infected with empty vector at both the mRNA and protein levels. (DI-D3) We found that in the RELM $\beta$-overexpressing cell strain, the inflammatory factors IL-8 and IL-I $\beta$ were expressed at higher levels than those in the parental and null control cell strains at both the mRNA and protein levels. (EI-E3) We successfully knocked down RELM $\beta$ gene expression; even under stimulation with tobacco smoke extract, the expression of RELM $\beta$ at both the mRNA and protein levels was lower than that in the tobacco smoke extract treatment group. (FI, F2) We found that the expression of the inflammatory factors IL-8 and IL-I $\beta$ was significantly downregulated after successful knock down of RELM $\beta$ gene expression. ${ }^{*} \mathrm{p} \leq 0.05, * * p \leq 0.0 \mathrm{I}, * * * p \leq 0.00 \mathrm{I}, * * * * p \leq 0.000 \mathrm{I}$. 
To further investigate the regulatory effect of RELM $\beta$ on the inflammatory factors IL-8 and IL- $1 \beta$, we decreased the gene expression of RELM $\beta$ by gene interference. After knocking down the RELM $\beta$ gene, even under stimulation with cigarette smoke extract, the expression of RELM $\beta$ was significantly decreased at both the mRNA and protein levels compared to the exposure to tobacco smoke extract alone (Figure 3E1E3). This result indicates that we successfully decreased the gene expression of RELM $\beta$. The expression of the inflammatory factors IL- 8 and IL-1 $\beta$ was significantly higher in the tobacco smoke extract stimulation group than in the parental control group. However, after successfully decreasing with RELM $\beta$ gene expression, the expression of these factors was significantly downregulated compared to control cells stimulated with tobacco smoke extract alone (Figure 3F1 and F2), indicating that RELM $\beta$ can regulate the expression of the inflammatory factors IL- 8 and IL-1 $\beta$. Our results suggest that RELM $\beta$ promotes inflammatory effects at both the mRNA and protein levels.

\section{RELM $\beta$ Promotes the Activation of the Phosphorylated p38 MAPK Signaling \\ Pathway}

To further investigate the signaling pathways involved the regulation of RELM $\beta$ expression during the airway inflammation associated with COPD, we analyzed the extracellular regulated protein kinase 1/2(ERK1/2), serine/threonine protein kinase $\mathrm{B}$ (PKB, also known as AKT) AKT, stress-activated protein kinase/stressactivated protein kinase (SAPK/JNK), and p38 mitogenactivated protein kinase (p38 MAPK) signaling pathway activation in response to RELM $\beta$. Our analysis showed that RELM $\beta$ could not activate the ERK1/2, AKT, and SAPK/JNK signaling pathways but could activate the phosphorylated p38 MAPK signaling pathway. In the stable RELM $\beta$ overexpression group, the expression of p-p38 MAPK was significantly higher than that in the parental and null control groups (Figure 4D1 and D2). When we successfully knocked down RELM $\beta$ gene expression, p38 MAPK could not be activated even in response to stimulation with tobacco smoke extract (Figure 4E1 and E2). Our results suggest that RELM $\beta$ activates the phosphorylated p38 MAPK signaling pathway, thereby promoting airway inflammation.

\section{RELM $\beta$ Promotes IL-8 and IL-I $\beta$ Expression via the p38 MAPK Signaling Pathway}

There is increasing evidence that the p38 MAPK signaling pathway is involved in inflammatory effects. ${ }^{21,22}$ To further analyze the potential mechanism by which RELM $\beta$ regulates the p38 MAPK signaling pathway, we treated the $16 \mathrm{HBE}$ cell line with the p38 MAPK signaling pathway inhibitor sb203580 for two hours. We analyzed the expression of phosphorylated p38 MAPK and the inflammatory factors IL-8 and IL-1 $\beta$. Our results showed that after we added increasing concentrations of the sb203580 inhibitor, phosphorylated p38 MAPK was significantly inhibited in the stable REALM-overexpressing cells (Figure 5A). We also observed that the expression of the inflammatory factors IL-8 and IL-1 $\beta$ was significantly decreased in the overexpression group treated with increasing concentrations of sb203580 (Figure 5A and B). Our results suggest that RELM $\beta$ activates phosphorylated p38 MAPK, promoting the expression of the inflammatory factors IL-8 and IL$1 \beta$ and participating in inflammatory effects.

\section{Discussion}

Inflammatory injury is the most important and central aspect of the pathogenesis and progression of chronic obstructive pulmonary disease. The most important feature of chronic obstructive pulmonary disease is the infiltration of multiple inflammatory cells into the airway wall and the obstruction of the lumen by increased airway mucous secretions, which cause airway obstruction and airway wall remodeling. ${ }^{23-25}$ Studies of the role of RELM $\beta$ in lung disease were initially focused on pulmonary fibrosis and pulmonary hypertension. ${ }^{10}$ Further studies of airway remodeling associated with pulmonary fibrosis and pulmonary hypertension showed that RELM $\beta$ expression was always accompanied by inflammatory effects. We then hypothesized that RELM $\beta$ likely acts as an inflammatory factor that mediates the regulatory network of chronic airway inflammation in COPD. Our data analysis showed that RELM $\beta$ is involved in regulating the network associated with airway inflammatory factors.

Analysis from our GEO database showed that RELM $\beta$ mRNA was significantly more highly expressed in the tissues of patients with COPD than in normal controls in two different datasets. Additionally, RELM $\beta$, a secretory 


\section{A1}

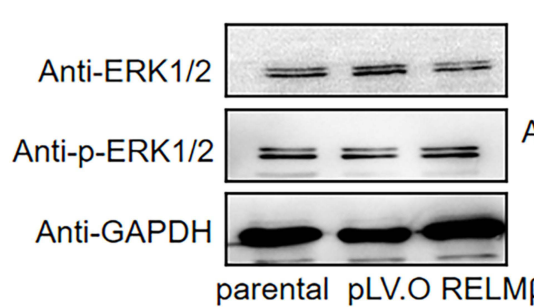

B1

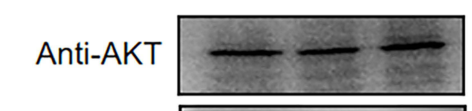

Anti-p-AKT(s473)

Anti-GAPDH

parental pLV.O RELM $\beta-O E$
C1

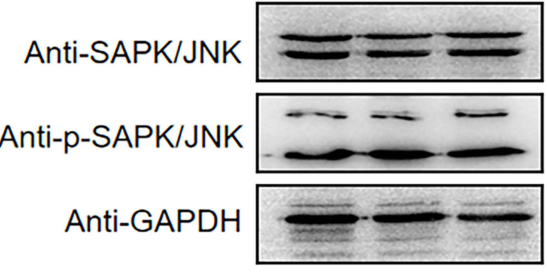

parental pLV.O RELM $\beta-O E$
A2

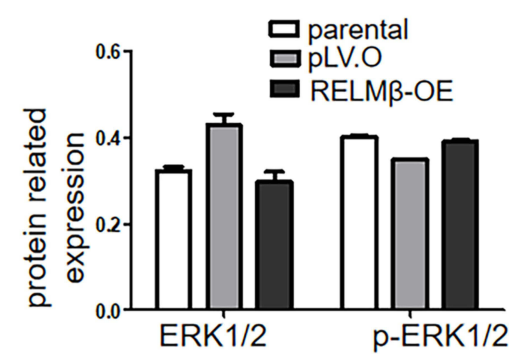

B2

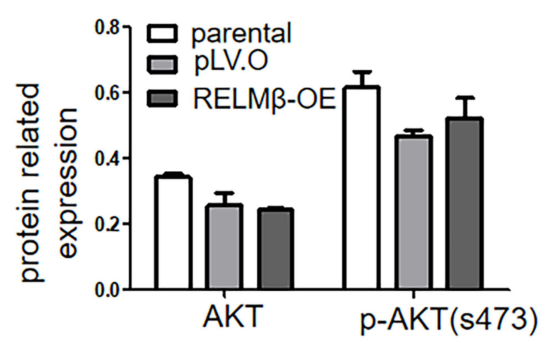

C2

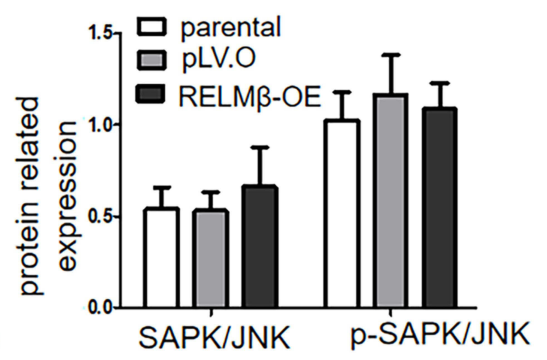

\section{D1}

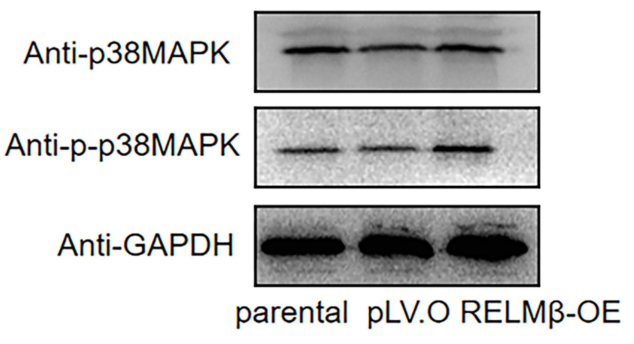

D2

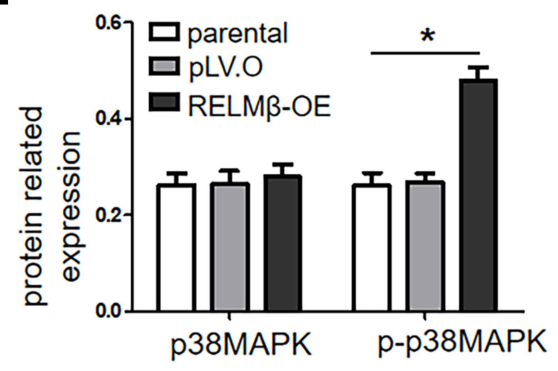

E1

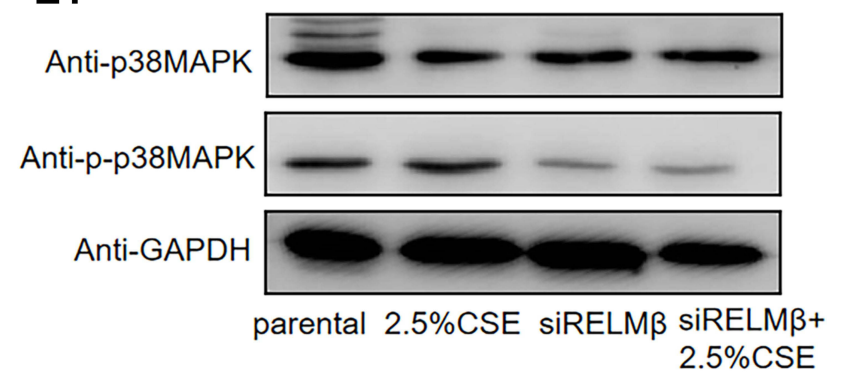

E2

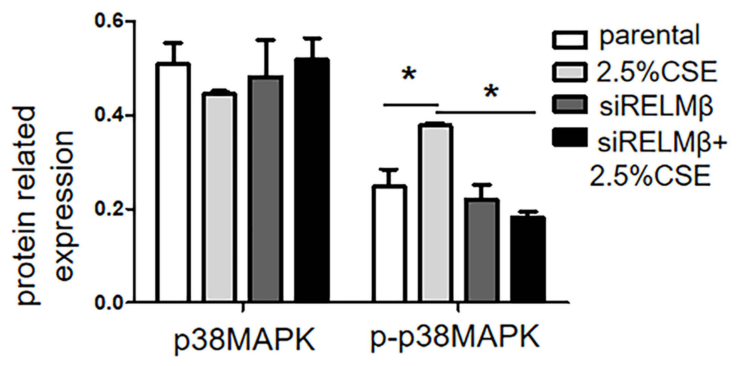

Figure 4 RELM $\beta$ activates the phosphorylated p38 MAPK signaling pathway. (A-C) Western blotting results showed that stable overexpression of RELM $\beta$ in I6HBE cells could not induce the phosphorylation of ERKI/2, Akt, and SAPK/JNK. (DI, D2) Our Western blotting results showed that RELM $\beta$ overexpression could induce phosphorylated $\mathrm{p} 38$ MAPK expression. (EI, E2) When we knocked down RELM $\beta$ gene expression, phosphorylated p38 MAPK was still not activated even after stimulation with tobacco smoke extract. *p $\leq 0.05$.

protein, is detectable in peripheral blood. ${ }^{9}$ Our ELISA results showed that the protein expression of RELM $\beta$ was also higher in the sera of patients with COPD than in that of controls. Our experiments suggest that RELM $\beta$ plays an essential role in the pathological progression of COPD. Further protein interaction network analysis showed that RELM $\beta$ has a significant regulatory relationship with IL-4, IL-10, and IL-8, which are closely related to airway inflammation regulation. These inflammatory factors involved in regulation were mainly enriched in the signaling pathway of cytokine interactions. These results suggest that RELM $\beta$ is involved in regulating networks that are enriched primarily in the regulation of inflammation-related signaling pathways. 


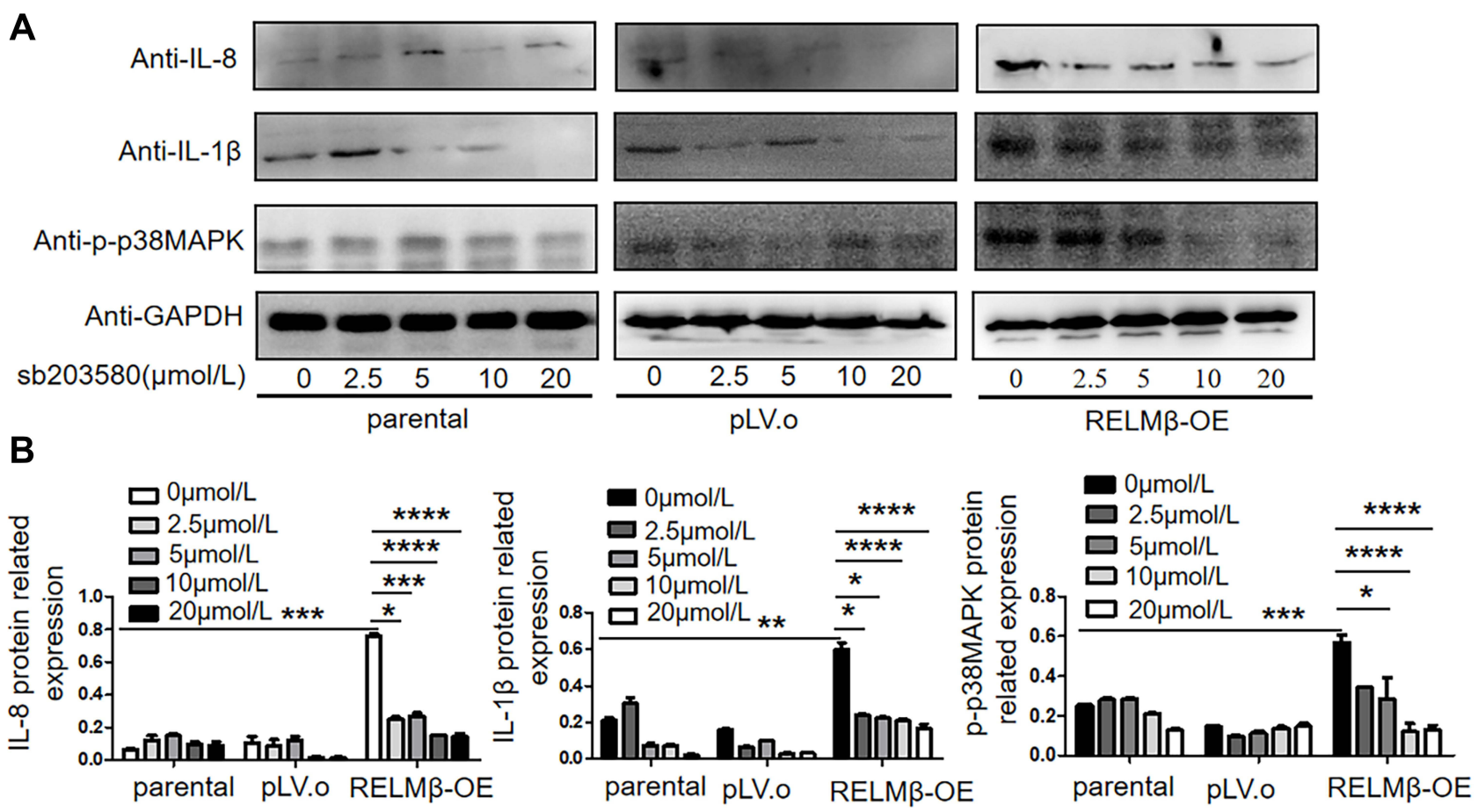

Figure 5 RELM $\beta$ promotes the expression of the inflammatory factors IL- 8 and IL-I $\beta$ via the $\mathrm{p} 38$ MAPK signaling pathway. (A-B) Western blotting and bar graphs showing the expression of $\mathrm{p}-\mathrm{p} 38 \mathrm{MAPK}, \mathrm{IL}-8$, and IL- $\mathrm{I} \beta$ in the $16 \mathrm{HBE}$ cells in the parental control, null and RELM $\beta$ overexpression groups after treatment with the $\mathrm{p} 38$ MAPK inhibitor sb203580. * $p \leq 0.05$, ** $p \leq 0.01$, *** $p \leq 0.001$, **** $p \leq 0.000$ I.

MAPK plays a key feedback role in airway inflammation by controlling the extent and duration of proinflammatory molecules. ${ }^{26}$ RELM $\beta$ activates the MAPK pathway, which results in increased levels of phosphorylated ERK1/ 2, p38 MAPK, and JNK, thereby activating the nuclear transcription factor NF- $\mathrm{KB}$ to lead to the secretion of various cytokines to exert further biological effects. ${ }^{27}$ After treating pulmonary arterial smooth muscle cells with exogenous recombinant RELM $\beta$ protein, the levels of phosphorylated MAPK and PKC increased, which was accompanied by a significant increase in cell viability. ${ }^{14}$ It has been shown that erythromycin inhibits p38 MAPK phosphorylation in patients with chronic obstructive pulmonary disease, thereby improving sensitivity to glucocorticoids and reducing the level of inflammation in patients with chronic obstructive pulmonary disease. ${ }^{28}$ The studies described above suggest that RELM $\beta$, together with the MAPK signaling pathway, plays a vital role in regulating airway inflammation. To further validate the involvement of RELM $\beta$ in the regulation of MAPK signaling pathways, we constructed a stable cell strain in which RELM $\beta$ was overexpressed to analyze the principle of the MAPK signaling pathway. Our results show that RELM $\beta$ stably activates p38 MAPK phosphorylation in the airway epithelium. In addition, we also investigated the ERK1/2, JNK, and AKT signaling pathways and showed that RELM $\beta$ could not activate those signaling pathways. This is different from signaling pathways, such as p38 MAPK and JNK, that RELM $\beta$ can initiate in human thylakoid cells ${ }^{29}$ and intestinal epithelial cells, ${ }^{30}$ suggesting that there are differences in the signaling regulatory pathways regulated by RELM $\beta$ in different tissues.

IL-8, the strongest chemokine produced by neutrophils, plays a significant role in the late stage of airway inflammation in COPD. ${ }^{31,32}$ IL-8 can induce neutrophils to migrate towards the site of inflammation, thus increasing the burden of the inflammatory site. Other inflammatory factors released from the inflammatory site, such as TNF- $\alpha$ and $\gamma$ IFN, can damage vascular endothelial cells and affect the release of oxygen radicals and nitrogen groups, thereby mediating the further release of inflammatory factors, amplifying the inflammatory effect and promoting inflammatory injury. ${ }^{33,34}$ In addition, IL-1 $\beta$, a more powerful proinflammatory cytokine, is a potent inducer of IL- 8 and IL-6 secretion by bronchial epithelial cells. In addition, IL-1 $\beta$ promotes increased numbers of $\mathrm{T}$ lymphocytes and dendritic cells in alveolar lavage fluid and lung tissue, and further analysis has shown similar results in COPD. ${ }^{35-38}$ The 
activation of inflammatory cells, such as macrophages and neutrophils, can lead to a significant increase in the serum IL-1 $\beta$ levels, resulting in further activation of macrophages, which promotes the release of inflammatory factors, such as IL-8, $\gamma \mathrm{IFN}$, and IL-12, exacerbating the local inflammatory response and thus leading to structural lung injury. ${ }^{39}$ In an ozone-induced airway inflammation mouse model, $\mathrm{Fe}^{40}$ and other investigators found that serum levels of inflammatory factors, such as IL-1 $\beta$, IL- 8 , and IL- 6 , the activity of NF- $\kappa$ B and p38 MAPK, and the density of airway inflammatory factor infiltration were significantly higher in the experimental group, while the FEV25 levels were substantially lower. Some domestic researchers have shown that elevated levels of p38 MAPK phosphorylation in the sputum of patients with COPD significantly correlate with high levels of IL-8, and the extent of this elevation substantially correlates with increased neutrophil infiltration and decreased lung function in patients. ${ }^{41}$ Our study found that RELM $\beta$ activates p-p38 MAPK and that the p38 MAPK inhibitor sb203580 decreases the expression of the inflammatory factors IL- 8 and IL-1 $\beta$. This inhibition was exacerbated with increasing concentrations of the inhibitor. Our results suggest that $\mathrm{p} 38$ MAPK is a downstream target molecule of RELM $\beta$ mediated airway inflammation. The p38 MAPK signaling pathway is necessary for the promotion of airway inflammation by RELM $\beta$ in COPD via IL- 8 and IL- $1 \beta$.

In conclusion, our results showed that RELM $\beta$ promoted the expression of the inflammatory mediators IL-8 and IL-1 $\beta$ in bronchial epithelial cells and that activation of the p38 MAPK signaling pathway was involved in RELM $\beta$-mediated airway inflammation. These results suggest that RELM $\beta$ may be a potential therapeutic target for patients with chronic obstructive pulmonary disease.

\section{Data Sharing Statement}

All publications discussed in the manuscript are available from the corresponding author on request.

\section{Statement of Ethics}

This study was approved by an institutional review board from the First Affiliated Hospital of Jinan University (Approval number: KY-2021-051) and conducted in accordance with the Declaration of Helsinki. The 16HBE bronchial epithelial cells was also approved by an institutional review board from the First Affiliated Hospital of Jinan University (Approval number: KYk-2021-017). All patients provided written informed consent in this study.

\section{Author Contributions}

All authors made substantial contributions to conception and design, acquisition of data, or analysis and interpretation of data. We agreed to submit to the current journal, gave final approval of the version to be published, and agreed to be accountable for all aspects of the work.

\section{Disclosure}

The authors declare that they have no financial or nonfinancial conflicts of interest for this work.

\section{References}

1. Halpin D, Criner G, Papi A, et al. Global initiative for the diagnosis, management, and prevention of chronic obstructive lung disease. The 2020 GOLD science committee report on COVID-19 and chronic obstructive pulmonary disease. Am J Respir Crit Care Med. 2021;203 (1):24-36. doi:10.1164/rccm.202009-3533SO

2. Zhou M, Wang H, Zeng X, et al. Mortality, morbidity, and risk factors in China and its provinces, 1990-2017: a systematic analysis for the Global Burden of Disease Study 2017. 2019;394 (10204):1145-1158. doi:10.1016/s0140-6736(19)30427-1

3. Barnes P. COPD 2020: new directions needed. Am J Physiol Lung Cell Mol Physiol. 2020;319(5):L884-L886. doi:10.1152/ajplung.00 473.2020

4. Hogg JC. Pathophysiology of airflow limitation in chronic obstructive pulmonary disease. Lancet. 2004;364(9435):709-721.

5. Liu S, Zhou Y, Wang X, et al. Biomass fuels are the probable risk factor for chronic obstructive pulmonary disease in rural South China. Thorax. 2007;62(10):889-897.

6 . Hogg J, Timens W. The pathology of chronic obstructive pulmonary disease. Anпu Rev Pathol. 2009;4:435-459. doi:10.1146/annurev. pathol.4.110807.092145

7. Holcomb I, Kabakoff R, Chan B, et al. FIZZ1, a novel cysteine-rich secreted protein associated with pulmonary inflammation, defines a new gene family. EMBO J. 2000;19(15):4046-4055. doi:10.1093/ emboj/19.15.4046

8. Banerjee A, Mondal NK, Das D, Ray MR. Neutrophilic inflammatory response and oxidative stress in premenopausal women chronically exposed to indoor air pollution from biomass burning. Inflammation. 2012;35(2):671-683.

9. Steppan C, Brown E, Wright C, et al. A family of tissue-specific resistin-like molecules. Proc Natl Acad Sci U S A. 2001;98 (2):502-506. doi:10.1073/pnas.98.2.502

10. Liu T, Baek H, Yu H, et al. FIZZ2/RELM- $\beta$ induction and role in pulmonary fibrosis. J Immunol. 2011;187(1):450-461. doi:10.4049/ jimmunol.1000964

11. Fang C, Yin L, Sharma S, et al. Resistin-like molecule- $\beta$ (RELM- $\beta$ ) targets airways fibroblasts to effect remodelling in asthma: from mouse to man. Clin Exp Allergy. 2015;45(5):940-952. doi:10.1111/cea.12481

12. Yamaji-Kegan K, Su Q, Angelini D, Myers A, Cheadle C, Johns R. Hypoxia-induced mitogenic factor (HIMF/FIZZ1/RELMalpha) increases lung inflammation and activates pulmonary microvascular endothelial cells via an IL-4-dependent mechanism. $J$ Immunol. 2010;185(9):5539-5548. doi:10.4049/jimmunol.0904021

13. Angelini D, Su Q, Yamaji-Kegan K, et al. Resistin-like molecule-beta in scleroderma-associated pulmonary hypertension. Am J Respir Cell Mol Biol. 2009;41(5):553-561. doi:10.1165/rcmb.2008-0271OC

14. Tian $\mathrm{H}$, Liu $\mathrm{L}$, Wu $\mathrm{Y}$, et al. Resistin-like molecule $\beta$ acts as a mitogenic factor in hypoxic pulmonary hypertension via the Cadependent PI3K/Akt/mTOR and PKC/MAPK signaling pathways. Respir Res. 2021;22(1):8. doi:10.1186/s12931-020-01598-4 
15. Lin C, Chen L, Huang Z, Wu Y, Liu S. Effect of cigarette smoke extraction on the expression of found in inflammatory zone 1 in rat lung epithelial L2 cells. Chin Med J. 2014;127(12):2363-2367.

16. Johns R, Takimoto E, Meuchel L, et al. Hypoxia-inducible factor $1 \alpha$ is a critical downstream mediator for hypoxia-induced mitogenic factor (FIZZ1/RELM $\alpha$ )-induced pulmonary hypertension. Arterioscler Thromb Vasc Biol. 2016;36(1):134-144. doi:10.1161/atvbaha.115.306710

17. Propheter D, Chara A, Harris T, Ruhn K, Hooper L. Resistin-like molecule $\beta$ is a bactericidal protein that promotes spatial segregation of the microbiota and the colonic epithelium. Proc Natl Acad Sci U S A. 2017;114(42):11027-11033. doi:10.1073/pnas.1711395114

18. Tsuboi K, Nishitani M, Takakura A, Imai Y, Komatsu M, Kawashima H. Autophagy protects against colitis by the maintenance of normal gut microflora and secretion of mucus. J Biol Chem. 2015;290(33):20511-20526. doi:10.1074/jbc.M114.632257

19. Wernstedt Asterholm I, Kim-Muller J, Rutkowski J, Crewe C, Tao C, Scherer P. Pathological Type-2 immune response, enhanced tumor growth, and glucose intolerance in Retnl $\beta$ (RELM $\beta$ ) null mice: a model of intestinal immune system dysfunction in disease susceptibility. Am J Pathol. 2016;186(9):2404-2416. doi:10.1016/j.ajpath.2016.04.017

20. Barnes P. Immunology of asthma and chronic obstructive pulmonary disease. Nat Rev Immunol. 2008;8(3):183-192. doi:10.1038/nri2254

21. Zhou R, Qu Y, Huang Q, Sun X, Mu D, Li X. Recombinant CC16 regulates inflammation, oxidative stress, apoptosis and autophagy via the inhibition of the p38MAPK signaling pathway in the brain of neonatal rats with sepsis. Brain Res. 2019;1725:146473. doi:10.1016/ j.brainres.2019.146473

22. Lin Z, Jin J, Shan X. Fish oils protects against cecal ligation and puncture-induced septic acute kidney injury via the regulation of inflammation, oxidative stress and apoptosis. Int $\mathrm{J} \mathrm{Mol} \mathrm{Med}$. 2019;44(5):1771-1780. doi:10.3892/ijmm.2019.4337

23. Koo H, Vasilescu D, Booth S, et al. Small airways disease in mild and moderate chronic obstructive pulmonary disease: a cross-sectional study. Lancet Respir Med. 2018;6(8):591-602. doi:10.1016/s2213-2600(18)30196-6

24. Pirina P, Foschino Barbaro M, Paleari D, Spanevello A. Small airway inflammation and extrafine inhaled corticosteroids plus long-acting beta-agonists formulations in chronic obstructive pulmonary disease. Respir Med. 2018;143:74-81. doi:10.1016/j.rmed.2018.08.013

25. Hogg J, Chu F, Utokaparch S, et al. The nature of small-airway obstruction in chronic obstructive pulmonary disease. $N$ Engl $J$ Med. 2004;350(26):2645-2653. doi:10.1056/NEJMoa032158

26. Moosavi S, Prabhala P, Ammit A. Role and regulation of MKP-1 in airway inflammation. Respir Res. 2017;18(1):154. doi:10.1186/ s12931-017-0637-3

27. Kushiyama A, Shojima N, Ogihara T, et al. Resistin-like molecule beta activates MAPKs, suppresses insulin signaling in hepatocytes, and induces diabetes, hyperlipidemia, and fatty liver in transgenic mice on a high fat diet. J Biol Chem. 2005;280(51):42016-42025. doi:10.1074/jbc.M503065200

28. Bin Y, Ma N, Lu Y, et al. Erythromycin reverses cigarette smoke extract-induced corticosteroid insensitivity by inhibition of the JNK/ c-Jun pathway. Free Radic Biol Med. 2020;152:494-503. doi:10. 1016/j.freeradbiomed.2019.11.020
29. Wang Y, Fan C, Chen B, Shi J. Resistin-like molecule beta (RELM- $\beta$ ) regulates proliferation of human diabetic nephropathy mesangial cells via Mitogen-Activated Protein Kinases (MAPK) signaling pathway. Med Sci Monitor. 2017;23:3897-3903. doi:10.12659/msm.905381

30. Chen G, Wang S, Jang J, Odegaard J, Nair M. Comparison of RELM $\alpha$ and RELM $\beta$ single- and double-gene-deficient mice reveals that RELM $\alpha$ expression dictates inflammation and worm expulsion in Hookworm infection. Infect Immun. 2016;84(4):1100-1111. doi:10. 1128/iai.01479-15

31. Higham A, Bostock D, Booth G, Dungwa J, Singh D. The effect of electronic cigarette and tobacco smoke exposure on COPD bronchial epithelial cell inflammatory responses. Int J Chron Obstruct Pulmon Dis. 2018;13:989-1000. doi:10.2147/copd.S157728

32. Zeng Y, Hu W, Zuo Y, Wang X, Zhang J. Altered serum levels of type I collagen turnover indicators accompanied by IL-6 and IL-8 release in stable COPD. Int J Chron Obstruct Pulmon Dis. 2019;14:163-168. doi:10.2147/copd.S188139

33. Hogan S, Seidu L, Blanchard C, et al. Resistin-like molecule beta regulates innate colonic function: barrier integrity and inflammation susceptibility. J Allergy Clin Immunol. 2006;118(1):257-268. doi:10. 1016/j.jaci.2006.04.039

34. Keatings V, Collins P, Scott D, Barnes P. Differences in interleukin- 8 and tumor necrosis factor-alpha in induced sputum from patients with chronic obstructive pulmonary disease or asthma. Am J Respir Crit Care Med. 1996;153(2):530-534. doi:10.1164/ajrccm.153.2.8564092

35. Khan Y, Kirkham P, Barnes P, Adcock I. Brd4 is essential for IL-1 $\beta$ induced inflammation in human airway epithelial cells. PLoS One. 2014;9(4):e95051. doi:10.1371/journal.pone.0095051

36. Ozretić P, da Silva Filho M, Catalano C, et al. NLRP1Association of coding polymorphism with lung function and serum IL-1 $\beta$ concentration in patients diagnosed with chronic Obstructive Pulmonary Disease (COPD). Genes. 2019;10:10. doi:10.3390/genes10100783

37. Fu J, McDonald V, Baines K, Gibson P. Airway IL-1 $\beta$ and systemic inflammation as predictors of future exacerbation risk in asthma and COPD. Chest. 2015;148(3):618-629. doi:10.1378/chest.14-2337

38. Osei E, Brandsma C, Timens W, Heijink I, Hackett T. Current perspectives on the role of interleukin-1 signalling in the pathogenesis of asthma and COPD. Eur Respir J. 2020;55:2. doi:10.1183/ 13993003.00563-2019.

39. Birrell M, Eltom S. The role of the NLRP3 inflammasome in the pathogenesis of airway disease. Pharmacol Ther. 2011;130(3): 364-370. doi:10.1016/j.pharmthera.2011.03.007

40. Fei X, Bao W, Zhang $\mathrm{P}$, et al. Inhalation of progesterone inhibits chronic airway inflammation of mice exposed to ozone. Mol Immunol. 2017;85:174-184. doi:10.1016/j.molimm.2017.02.006

41. Huang C, Xie M, He X, Gao H. Activity of sputum p38 MAPK is correlated with airway inflammation and reduced FEV1 in COPD patients. Med Sci Monit. 2013;19:1229-1235. doi:10.12659/MSM. 889880

International Journal of Chronic Obstructive Pulmonary Disease

Dovepress

\section{Publish your work in this journal}

The International Journal of COPD is an international, peer-reviewed journal of therapeutics and pharmacology focusing on concise rapid reporting of clinical studies and reviews in COPD. Special focus is given to the pathophysiological processes underlying the disease, intervention programs, patient focused education, and self management protocols. This journal is indexed on PubMed Central, MedLine and CAS. The manuscript management system is completely online and includes a very quick and fair peer-review system, which is all easy to use. Visit http://www.dovepress.com/testimonials.php to read real quotes from published authors. 